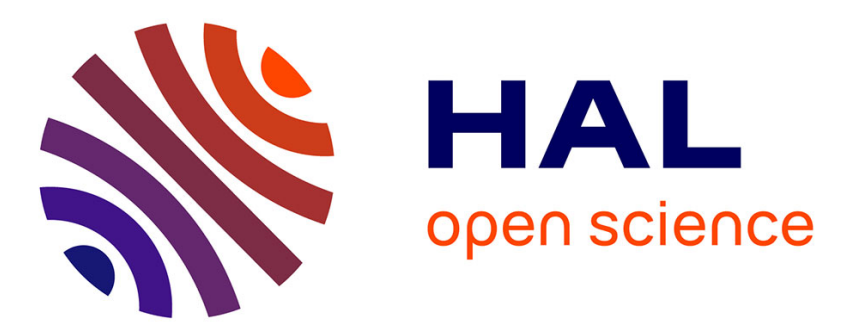

\title{
Evaluation of halogen chain-end functionality in 2-bromo-2-methylpropanoate esters of poly(oxyalkylene) polymers by MALDI-TOF spectroscopy
}

Angélica Velázquez-Arellano, Daniel Grande, Luis Ernesto Elizalde-Herrera

\section{- To cite this version:}

Angélica Velázquez-Arellano, Daniel Grande, Luis Ernesto Elizalde-Herrera. Evaluation of halogen chain-end functionality in 2-bromo-2-methylpropanoate esters of poly(oxyalkylene) polymers by MALDI-TOF spectroscopy. Polymer Bulletin, 2021, 78 (10), pp.5641-5665. 10.1007/s00289-02003391-1 . hal-03338590

\author{
HAL Id: hal-03338590 \\ https://hal.science/hal-03338590
}

Submitted on 8 Sep 2021

HAL is a multi-disciplinary open access archive for the deposit and dissemination of scientific research documents, whether they are published or not. The documents may come from teaching and research institutions in France or abroad, or from public or private research centers.
L'archive ouverte pluridisciplinaire HAL, est destinée au dépôt et à la diffusion de documents scientifiques de niveau recherche, publiés ou non, émanant des établissements d'enseignement et de recherche français ou étrangers, des laboratoires publics ou privés. 
1 Evaluation of halogen chain-end functionality in 2-bromo-

2 2-methylpropanoate esters of poly(oxyalkylene) polymers

3 by MALDI-TOF spectroscopy

\author{
4 Angélica Velázquez-Arellano, ${ }^{1}$ Daniel Grande, ${ }^{2}$ Luis Ernesto Elizalde-Herrera, ${ }^{1 *}$ \\ $5 \quad{ }^{1}$ Centro de Investigación en Química Aplicada (CIQA), Boulevard Enrique Reyna Hermosillo N ${ }^{\circ}$ 140. C.P. \\ 6 25253. Saltillo, Coahuila, Mexico. \\ 7 2'Institut de Chimie et des Matériaux Paris-Est (ICMPE), UMR 7182 CNRS-Université Paris-Est Créteil, 2 rue \\ 8 Henri Dunant, 94320 Thiais, France \\ 9 *Corresponding author e-mail: $\underline{\text { luis.elizalde@ciqa.edu.mx }}$
}

12 Keywords

13 MALDI-TOF MS; end-functionalization; PEG-based macroinitiators

14 Abstract

15 Esters of 2-bromo-2-methylpropanoate of poly(oxyalkylene) polymers such as poly(ethylene glycol) 16 or $\alpha$-methyl poly(ethylene glycol) were prepared in high yields and characterized by spectroscopic 17 and chromatographic methods (NMR, FT-IR, mass spectroscopy and SEC). The halogen chain-end 18 group in the poly(oxyalkylene) bromine-terminated esters were characterized by MALDI-TOF MS. 19 The effect of the solvents (methanol or tetrahydrofuran) and the cationic agents such as silver 20 trifluoroacetate (AgTFA), silver trifluoromethanesulfonate (AgTFS) and sodium trifluoroacetate 21 (NaTFA) on the mass spectra was studied. Analysis of the mass spectra demonstrated that the analyte 22 was transformed to unsaturated (elimination), alkoxy or hydroxyl end groups (substitution) molecules 23 when silver cationic agents were used, these results were also supported by $1 \mathrm{H}$ NMR study. When 24 sodium salt was used as a cationic agent, well-defined bromine-terminated macromolecules were 25 successfully determined through MALDI-TOF MS. Well characterized esters of 2-bromo-2- 
1 methylpropanoate of poly(oxyalkylene) polymers could be used as ATRP macroinitiators for the

2 synthesis of a variety of polymeric architectures of interest as drug delivery bioconjugates.

3

\section{$4 \quad$ Introduction}

5 Poly(oxyalkylene) polymers are known as hydrophilic polymers, and the most representative are

6 poly(ethylene glycol) (PEG) and $\alpha$-methyl poly(ethylene glycol) (MPEG), these type of materials are

7 frequently used for the synthesis of new and complex materials with applications in several areas,

8 mainly due to their biocompatibility and biodegradability properties [1][2][3]. In addition, when these

9 polymers are end-modified with a suitable functional group, they can be used as macroinitiators in 10 the reversible-deactivated radical polymerization (RDRP) mechanisms, including nitroxide-mediated 11 radical polymerization (NMP), reversible addition-fragmentation chain transfer (RAFT) 12 polymerization, and atom transfer radical polymerization (ATRP), which are the most commonly 13 used polymerization methods to obtain hydrophilic or amphiphilic polymeric materials [4][5][6][7].

14 ATRP is a versatile and efficient RDRP technique for the preparation of macromolecules with 15 predetermined molar masses, narrow molar mass distributions, well-defined topologies, and high 16 degrees of chain-end functionality [8]. This is possible due to its specific catalytic process, where an 17 halide atom is required to react with the metal complex to generate a radical that can act as an initiator 18 in the ATRP process [9][10]. Thus, the quantification and identification of these halide groups are 19 critical when materials with specific characteristics are required [11].

20 Traditional techniques, such as nuclear magnetic resonance (NMR), Fourier Transform Infrared (FT21 IR) or size exclusion chromatography (SEC), could give inaccurate results in the characterization and 22 quantification of halogen end-groups, mainly due to the low intensity of the end-group signals 23 compared with the signal intensity of the main polymer chain. This drawback is magnified when the 24 molecular weight is increased and the end-group signals may be too small and indistinguishable when 25 compared with the noise, therefore it cannot be quantified correctly [12][13]. In addition, these 26 techniques, provide information related with the average of all macromolecules rather than a 27 distribution of the polymer size, an important issue in ATRP [14] .

28 Mass spectroscopy was addressed to solve these challenges through the development of soft 29 ionization techniques, such as matrix-assisted laser desorption/ionization-time of flight mass 30 spectrometry (MALDI-TOF MS). With this technique, the polymer mass spectra are obtained without 31 fragmentation, and the exact molar mass of a single macromolecule can be accurately determined 
1 [15]. In the MALDI process, the sample is ionized and transferred into the gas-phase, thus molecular

2 ions $\left([\mathrm{M}]^{+}\right)$can be analysed [16][17]. Through this technique, the number-average molar mass $\left(M_{\mathrm{n}}\right)$,

3 mass-average molar mass $\left(M_{\mathrm{w}}\right)$, degree of polymerization $(D P)$ and dispersity $(\nexists)$ can be determined

4 with high precision [18][19]. However, MALDI data are accurate and precise only for polymers with

5 low dispersity $(\nexists>1.2)$. Materials with higher than this value, discrete discrepancies between the

6 experimental and theoretical molar masses values could be registered [20].

7 In order to get an accurate MALDI analysis, the selection of the matrix is crucial, the right matrix

8 facilitating the transfer of energy to polymer chains during the ionization process. Commonly, metal

9 salts are added to improve the ionization [21]. To this purpose, the sample preparation is an overriding

10 step for collecting high-quality MALDI-TOF spectra. It is important to consider the solubility of all

11 components (polymer, matrix, and metal salt) in a suitable solvent. Once the solutions of these

12 materials are placed in the MALDI target plate, the solvent is evaporated, and a proper co-

13 crystallization is needed to assure the interaction between all components and promotes the optimum

14 ionization conditions.

15 However, there is controversy in the scientific community regarding the analysis of end16 functionalized polymers obtained by RDRP in the attempts of obtain telechelics materials, where it 17 could be misunderstanding in the assignation of the correct structures through MALDI analysis. 18 Different examples are found in the art, Lallana et al. [22], found in poly(propylene sulfides) 19 functionalized at the end with an ester groups analyzed by MALDI a decarboxyalkylation reaction 20 during the ionization process. Borman et al. [23] found in PMMA bromine end functionalized a loss 21 of methylbromide followed by cyclisation of the final two monomer repeat units to give a lactone end 22 group. Matyjaszewski et al. [24] reported the loss of $\mathrm{HBr}$ in poly(acrylates) obtained by ATRP. In 23 addition, PMMA obtained with ATRP it was found a loss of the chlorine atom as the end group during 24 the MALDI analysis with the use of silver as cation agent [25]. Couthouis et al. [26] reported on the 25 presence of the disproportionation products as the main structures found in MALDI spectra, in spite 26 of PMMA samples with low dispersity, first-order kinetics related to the monomer consumption, good 27 control in the molar masses, thus suggesting mistakes in the assigned polymer structures.

28 According to previous reports [27][28], it is accepted that the halogen atom (X) cannot be observed 29 due to the lability of $\mathrm{C}-\mathrm{X}$ covalent bonds, resulting from a hydro-halo elimination reaction during the 30 sample preparation or in the MALDI-TOF MS process. These reactions provide unsaturated chain31 end groups, which can be confused with an unsaturated terminated polymer that can be obtained by 
1 disproportionation during the synthesis of the macroinitiator by ATRP, leading in a doubtful quality

2 of the ATRP macroinitiators [29].

3 Some authors, like Ladavière et al. [30], employed MALDI-TOF MS for the analysis of bromine4 terminated polystyrene (PS-Br) and chlorine-terminated polystyrene (PS-CI) prepared via ATRP 5 using AgTFA or sodium iodide (NaI) as cationizing agents. Poor ionization was obtained with NaI, 6 because the signal-to-noise ratio was not significant enough to assign any signal. In contrast, when 7 AgTFA was used, unsaturated macromolecules were observed, produced from elimination of 8 hydrochloric acid $(\mathrm{HCl})$ or hydrobromic acid $(\mathrm{HBr})$. This phenomenon was attributed to the MALDI9 TOF process, including the ionization step. In addition, other species of minor intensity were 10 observed, and not fully identified. Tintaru et al. [27] also analyzed by MALDI-TOF PS-Br samples 11 prepared by ATRP, which was previously characterized by NMR and electrospray ionization-mass 12 spectroscopy (ESI-MS). They concluded that when the silver cation $\left(\mathrm{Ag}^{+}\right)$was mixed with the PS-Br 13 macromolecules, the elimination of the bromine atom occurred, thus generating macromolecules with 14 different end groups, depending on the solvent, solution composition, matrix, and metal salts used. 15 Nonetheless, in all the cases, the loss of the bromine functionality was observed. Similar results were 16 reported by Kim et al. [28] for PS-Br synthetized by ATRP and characterized with MALDI-TOF MS 17 using AgTFA, NaTFA, or silver pentafluoropropionate (AgPFP) as cationization agents and 18 tetrahydrofuran (THF), toluene, chloroform, acetone, methanol and ethanol as solvents. They found 19 some PS macromolecules with unsaturated terminations, hydroxyl $(-\mathrm{OH})$ and other functionalities at 20 the chain end of the polymers, attributed to reactions between the PS macromolecules and the solvent 21 or the employed cations. As stated before, the most accepted postulate is the loss of the halogen atom 22 at the polymer chain-end during the sample preparation. Recently, Altintas et al. [31] analyzed PS$23 \mathrm{Br}$ synthesized via activators regenerated by electron transfer (ARGET) atom transfer radical 24 polymerization (ATRP). These authors reported that the removal of halide group occurred during the 25 ionization process and molecular species with unsaturated and hydroxyl chain-end groups were mainly observed.

27 Here we report the characterization of chain end-functionalization of poly(oxyalkylene) bromine28 terminated esters by MALDI-TOF MS. Six poly(oxyalkylene) polymers with a molecular weight 29 range between 700-7,000 Da were esterified with 2-bromo-2-methylpropionyl bromide and the 30 correspondent esters were purified and characterized by spectroscopic techniques. The effect of the 31 analyte molecular weight, solvents (methanol or tetrahydrofuran) and the cationic agents such as 32 silver trifluoroacetate (AgTFA), silver trifluoromethanesulfonate (AgTFS) and sodium trifluoroacetate (NaTFA) on the mass spectra was studied. 
Experimental

Materials. Powders of poly(ethylene glycol) (2000, 3350 and $6000 \mathrm{Da})$ and poly(ethylene glycol) monomethyl ether (750 (as wax), 2000 and $5000 \mathrm{Da}$ ) were purchased from Sigma-Aldrich and dried in a vacuum oven $(10 \mathrm{mmHg})$ at room temperature for 24 hours prior to use. Dichloromethane methanol $\left(\mathrm{CH}_{3} \mathrm{OH}, \mathrm{JTBK}, 97 \%\right)$, methanol- $d_{4} 99.8$ atom \% D ( $\mathrm{CD}_{3} \mathrm{OD}$, Aldrich,), trans-2-[3-(4-tertbutylphenyl)-2-methyl-2-propenylidene] malononitrile (DCTB, Aldrich,), silver

11 trifluoromethanesulfonate (AgTFS, Aldrich, 99\%), sodium trifluoroacetate (NaTFA, Aldrich, 99\%), 12 and chloroform- $d 99.8$ atom \% D ( $\mathrm{CDCl}_{3}$ Aldrich, ) were used as received. Silver trifluoroacetate 13 (AgTFA) was prepared from aqueous silver nitrate and trifluoroacetic acid, the product being 14 evaporated and washed with methanol and diethyl ether and finally vacuum dried (1 $\mathrm{mmHg})$ 15 overnight at room temperature.

Bromine end-functionalization in poly(ethylene glycol) and $\alpha$-methyl poly(ethylene glycol). In a three-neck flask, $0.001 \mathrm{mmol} \mathrm{OH}-(\mathrm{PEG})_{\mathrm{n}}-\mathrm{OH}$ or $\mathrm{CH}_{3}-(\mathrm{PEG})_{\mathrm{n}}-\mathrm{OH}$ were dissolved in $80 \mathrm{~mL}$ dried $\mathrm{CH}_{2} \mathrm{Cl}_{2}$, then 0.006 (for PEG) or $0.003 \mathrm{mmol}$ (for MPEG) of TEA were added, mixed, and cooled to $0{ }^{\circ} \mathrm{C}$. After this step, 0.002 or $0.001 \mathrm{mmol}$ of BIBB respectively were dissolved in $20 \mathrm{~mL}$ of dry $\mathrm{CH}_{2} \mathrm{Cl}_{2}$ and added dropwise within a $1 \mathrm{~h}$-period under $\mathrm{N}_{2}$ to the reaction for $7 \mathrm{~h}$ at $40^{\circ} \mathrm{C}$. The reaction mixture was concentrated in a rotary evaporator and extracted twice, first with an aqueous $\mathrm{HCl}$

22 solution $5 \% \mathrm{v} / \mathrm{v}$, then with an aqueous $\mathrm{NaHCO}_{3}$ solution $(10 \% \mathrm{w} / \mathrm{v})$, and finally with $\mathrm{CH}_{2} \mathrm{Cl}_{2}$. The 23 organic layers were collected and dried over $\mathrm{MgSO}_{4}$.

Sample preparation for NMR Analysis. Solution A was prepared in a $5 \mathrm{~mL}$ sample vial, $20 \mathrm{mg}$ of

$\mathbf{2 d}$ was accurate weighted in $2 \mathrm{~mL}$ of tetradeuteromethanol $\left(\mathrm{CD}_{3} \mathrm{OD}\right)$ then, the vial was immersed in an ice bath for five minutes. Solution B was obtained when $28 \mathrm{mg}$ of AgTFA was accurate weighed in a $5 \mathrm{~mL}$ sample vial and dissolved in $2 \mathrm{~mL}$ of $\mathrm{CD}_{3} \mathrm{OD}$ after, the sample vial was placed in an ice

28 bath for five minutes. The final solution was obtained when $0.5 \mathrm{~mL}$ of the solutions $\mathrm{A}$ and $\mathrm{B}$ were 29 mixed and placed in a 5mm NMR tube, this sample was analyzed by NMR.

30 Analytical techniques. The polymer samples were characterized by size exclusion chromatography 31 (SEC) using a Waters Alliance chromatograph model 2695 equipped with a refractive index detector 
model 2414 and two columns Agilent PL gel $5 \mu \mathrm{m}$ Mixed C. The samples were dissolved in anhydrous THF (10 $\left.\mathrm{mg} \mathrm{mL}^{-1}\right)$, and the solutions were filtered through $0.2 \mu \mathrm{m}$ nylon membranes. THF was used as an eluent at a flow rate of $1 \mathrm{~mL} \mathrm{~min}^{-1}$ at $30^{\circ} \mathrm{C}$. The system was calibrated with a linear calibration curve built from polystyrene standards (1,350 to $1,997,000 \mathrm{Da}$ PS), and the correction for the related to changes in the hydrodynamic volumes was carried out with the Kuhn-Mark-HouwnikSakurada constants for the PEG-THF system $\left([\eta]=3.39 \times 10^{-4} \mathrm{M}^{0.66}\right)[32]$.

Proton nuclear magnetic resonance $\left({ }^{1} \mathrm{H}\right.$ NMR) spectra were generally recorded at room temperature $\left(298^{\circ} \mathrm{K}\right)$ in $\mathrm{CDCl}_{3}$ with TMS as an internal standard on a Bruker AVANCE III spectrometer (500 $\mathrm{MHz})$.

Fourier-transform infrared (FT-IR) spectra were performed on a Thermo-Nicolet iS10 spectrometer in transmittance mode with 64 scans and a spectral resolution of $2 \mathrm{~cm}^{-1}$ in the range from 4000 to 400 $\mathrm{cm}^{-1}$. The samples were analyzed on $\mathrm{KBr}$ pellet.

MALDI-TOF Mass Spectrometry. Matrix-assisted laser desorption/ionization-time of flight mass spectrometry (MALDI-TOF MS) were acquired on a Bruker autoflexTM speed mass spectrometer (Bruker Daltonics, Billerica Ma.). The system uses a frequency-tripled Nd:YAG laser (355 nm) and a $2 \mathrm{kHz}$ repetition rate (Smartbeam-II ${ }^{\mathrm{TM}}$ ). Measurements were carried out in reflectron mode (for sample with the mass range from 0.7 to $5 \mathrm{kDa}$ ) or linear mode (for sample with molar masses up to 5 $\mathrm{kDa}$ ). Positive-ion mode was used for all analyses with an acceleration voltage of $20.00 \mathrm{Kv}$ and delayed extraction. Ions in the mass range $(\mathrm{m} / \mathrm{z})$ of $700-7000$ were detected with a sampling rate of 5.0 GS s${ }^{-1}$ for reflectron mode and $0.63 \mathrm{GS} \mathrm{s}^{-1}$ for linear mode. The collected data were added with over 500 shots at a frequency of a $2000 \mathrm{~Hz}$; the laser probe was slowly moved to avoid poor signals. The laser power energy was tuned to yield sufficiently intense signals (60\% in linear mode and $80 \%$ reflectron mode). Insulin (5734.52 Da), cytochrome c (6161.05 Da), myoglobin (8476.66), ubiquitin I (8565.89 Da), cytochrome c (12360.97 Da), and myoglobin (16952.31 Da) were used as external calibrants enabling a mass accuracy of about $50 \mathrm{ppm}$. The resolution of the MALDI spectra reaches ranges between 2000 and 18000, depending on molar mass distribution, purity and cationic agent used.

Sample Preparation. PEG, MPEG, and their corresponding esters were dissolved in $\mathrm{CH}_{3} \mathrm{OH}$ or THF at a concentration of $5 \mathrm{mg} \mathrm{mL}^{-1}$. DCTB solution was prepared with a concentration of $20 \mathrm{mg} \mathrm{mL}^{-1}$. $0.1 \mathrm{M}$ solutions of cationization agents (AgTFS, AgTFA, NaTFA) were also prepared. Solutions of $10 \mu \mathrm{L}$ of the polymer, $10 \mu \mathrm{l}$ of DCTB, and $2.0 \mu \mathrm{l}$ cationization agents were mixed and deposited on a MALDI-TOF MS sample plate, and the spots were dried in air at room temperature before analysis. 
1 Data Analysis. Data acquisition was performed using flexControl software 3.4 (Bruker Daltonics,

2 Billerica Ma.) and data analysis the mass of each selected monoisotopic peak was determined using

3 a centroid algorithm calculated with FlexAnalysis software 3.4 (Bruker Daltonics, Billerica Ma.)

4 assuming PEG elemental composition. MS data were processed using PolyTools 1.31 (Bruker

5 Daltonics).

$6 \quad M_{\mathrm{n}}, M_{\mathrm{w}}$, and $\boldsymbol{\nexists}$ calculation with MALDI-TOF MS. $M_{\mathrm{n}}, M_{\mathrm{w}}$, and $Ð$ values for PEG, MPEG, and

7 their corresponding esters were calculated without software support. $M_{\mathrm{n}}$ was calculated as follows:

$8 \quad M_{\mathrm{n}}=\Sigma\left(\mathrm{n}_{\mathrm{i}} M_{\mathrm{i}}\right) / \Sigma \mathrm{n}_{\mathrm{i}}, \mathrm{n}_{\mathrm{i}}$ representing the intensity found in the MALDI spectra and $M_{\mathrm{i}}$ the $m / z$ ratio. $M_{\mathrm{w}}$

9 was calculated as follows: $M_{\mathrm{w}}=\Sigma\left(\mathrm{n}_{\mathrm{i}} M_{\mathrm{i}}^{2}\right) / \Sigma\left(\mathrm{n}_{\mathrm{i}} M_{\mathrm{i}}\right) . Ð$ was inferred from the resulting $M_{\mathrm{w}} / M_{\mathrm{n}}$ ratio.

\section{Results and Discussion}

12 PEG and MPEG Characterization. The molar masses of the six samples of poly(oxyalkylene) 13 polymers (1a-f) were determined by MALDI-TOF MS and compared with those obtained by NMR 14 and SEC, the results being listed in Table 1. The experimental peak values of molar masses $\left(M_{\mathrm{p}}\right)$ 15 were obtained from MALDI-TOF analysis, and they had the largest intensity in the observed 16 distributions of the mass spectra. Theoretical molar masses were calculated for PEG or MPEG considering the degree of polymerization $(D P)$ and hydroxyl $v s$. methyl substituents at $\alpha$ and $\omega$ positions. $M_{\mathrm{n}}, D P$, and $\oslash$ values were calculated from the mass spectra. The experimental values $M_{\mathrm{p}}$ were compared with the theoretical ones for the six poly(oxyalkylene) materials characterized by MALDI-TOF MS, and an excellent agreement between these values $(\Delta M \approx 1)$ was obtained.

21 Therefore, the molecular formula of these polymers can be established as $\mathrm{HO}\left(\mathrm{CH}_{2} \mathrm{CH}_{2} \mathrm{O}\right)_{n} \mathrm{H}$ for PEG and $\mathrm{CH}_{3} \mathrm{O}\left(\mathrm{CH}_{2} \mathrm{CH}_{2} \mathrm{O}\right)_{\mathrm{n}} \mathrm{H}$ for MPEG. When the MALDI results were compared with the molecular weight obtained with SEC, differences among them can be appreciated. In Table 1, higher molecular weight values were determined with SEC, in spite of the correction to the changes in the hydrodynamic volumes with the Kuhn-Mark-Houwnik-Sakurada constants for the PEG-THF system ([ๆ] =3.39x10-4M0.66). In addition, the average molecular weight was calculated by NMR which

27 shown better agreement with those determined with MALDI-TOF, its behavior could lead to suppose 28 an overestimation of the molecular weight with SEC analysis. With these results, can be stated that 29 the ion suppression phenomena it is absent during the MALDI analysis, due to the observed narrow dispersities $(\fallingdotseq<1.03)$ for PEG and MPEG polymers [33]. 

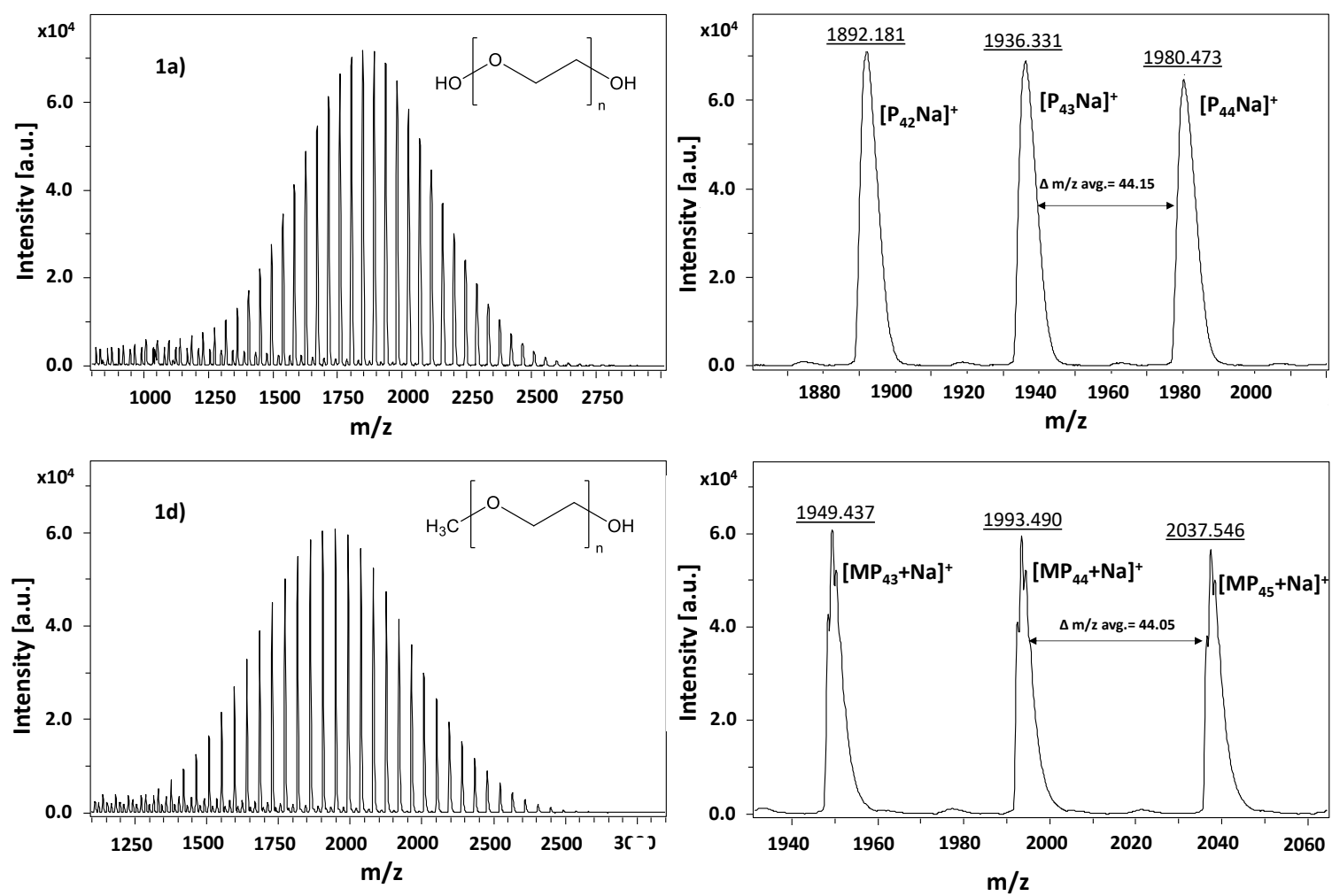

Figure 1. MALDI-TOF MS obtained in reflectron mode of PEG (1a) and MPEG (1d) in DCTB as a matrix and NaTFA as an ionic agent in THF.

4 Synthesis of ATRP macroinitiators from PEG and MPEG. Once the poly(glycol)s were 5 characterized, and with the aim to obtain end-functionalized polymers as ATRP macroinitiators, the 6 esterification of the poly(glycol)s were performed through their reaction with 2-bromo-27 methylpropanoyl bromide and triethylamine in dichloromethane for $7 \mathrm{~h}$ at $40^{\circ} \mathrm{C}$ (Scheme 1).

a) $\mathrm{R}$

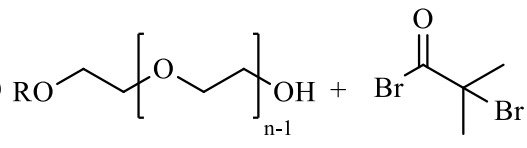

1a $\mathrm{R}=\mathrm{H}, \mathrm{n}=43 ; \mathbf{1 b} \mathrm{R}=\mathrm{H}, \mathrm{n}=74 ; \mathbf{1 c} \mathrm{R}=\mathrm{H}, \mathrm{n}=131$

1d $\mathrm{R}=\mathrm{CH}_{3}, \mathrm{n}=15 ; \mathbf{1 e} \mathrm{R}=\mathrm{CH}_{3}, \mathrm{n}=44 ; \mathbf{1 f} \mathrm{R}=\mathrm{CH}_{3}, \mathrm{n}=117$
8

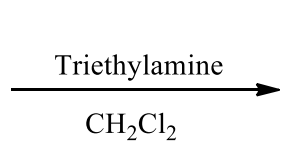

b) $\mathrm{R}_{1} \mathrm{O} \sim \mathrm{N}_{\mathrm{n}-1}^{\mathrm{O}} \overbrace{\mathrm{Br}}^{\mathrm{O}}$

$$
\text { 2a } \mathrm{R}_{1}=Z, \mathrm{n}=43 ; \mathbf{2 b} \mathrm{R}_{1}=Z, \mathrm{n}=74 ; \mathbf{2 c} \mathrm{R}_{1}=Z, \mathrm{n}=131
$$
2d $\mathrm{R}_{1}=\mathrm{CH}_{3}, \mathrm{n}=15 ; \mathbf{2 e} \mathrm{R}_{1}=\mathrm{CH}_{3}, \mathrm{n}=44 ; \mathbf{2} \mathbf{2} \mathrm{R}_{1}=\mathrm{CH}_{3}$, $\mathrm{n}=117$

$$
\mathrm{Z}=\stackrel{\mathrm{O}}{\mathrm{H}} \mathrm{Br}
$$

Scheme 1. PEG and MPEG esterification with 2-bromo-2-methylpropanoyl bromide.

The ester groups of poly(glycol)s (2a-f) were characterized via spectroscopic techniques and yields were gravimetrically calculated after the purification process. The spectroscopic characterization was focused on identifying the 2-bromo-2-methylpropanoate moiety in the esterified polymer samples. FT-IR analysis of the esters (2a-f) showed a stretching band at $1734 \mathrm{~cm}^{-1}$, characteristic of the ester 
1 group $(\mathrm{C}=\mathrm{O})$ (Figure $\mathrm{S}-1$ in Supporting Information). In the ${ }^{1} \mathrm{H}$ NMR spectra of the esters 2a-f, the

2 2-bromo-2-methylpropanoate moiety was identified as a triplet at $4.29 \mathrm{ppm}$, as it was a signal

3 characteristic of the methylene $\left(-\mathrm{CH}_{2}\right)$ adjacent to the oxygen atom in the ester group. In addition,

4 the signal for the $\alpha$-methyl groups of the ester moiety had a chemical shift of 1.85 ppm (Figure 2).

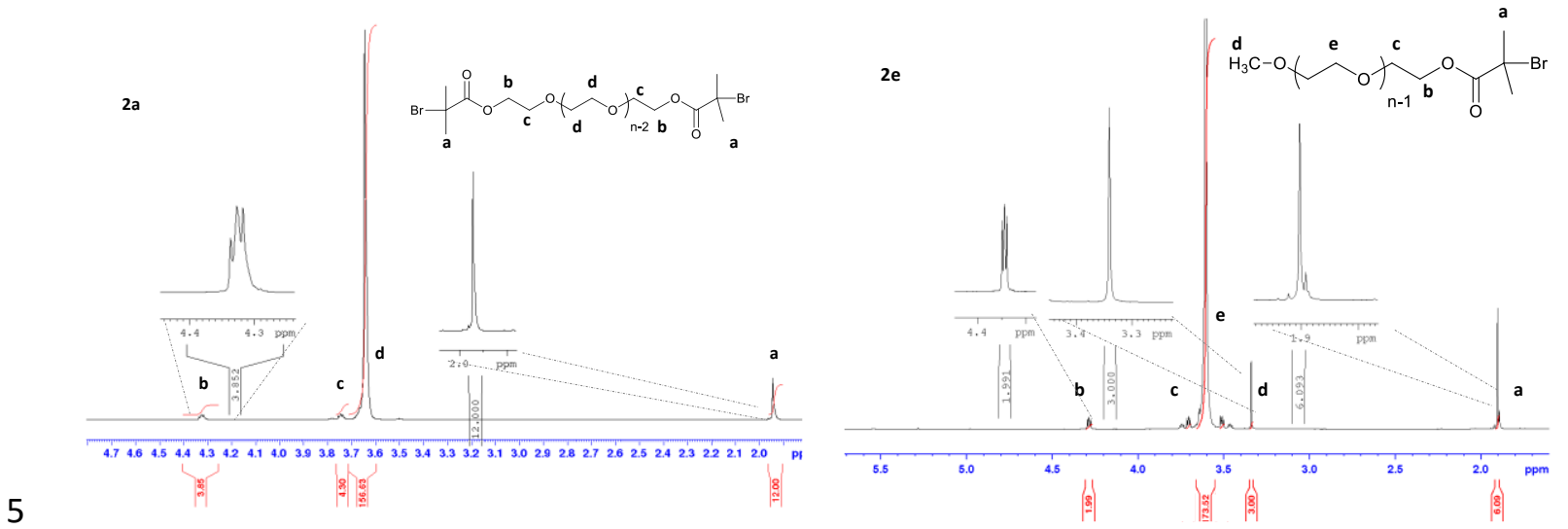

6 Figure 2. ${ }^{1} \mathrm{H}$ NMR $(500 \mathrm{MHz})$ spectra of $\mathbf{2 a}$ and $\mathbf{2 e}$ macroinitiators in $\mathrm{CDCl}_{3}$.

7 Once the chemical structure was established, the ester functionalization degree was calculated from

8 the integration ratio of the signals associated with characteristic protons. Indeed, the signal at 1.85

9 ppm for the methyl of the ester group and the band at $3.37 \mathrm{ppm}$ for the methoxy group were taken as

10 reference for the MPEG samples, while the band at $1.85 \mathrm{ppm}$ and the ethylene resonances at 3.63

11 ppm were considered for the PEG polymers. The values demonstrated a near quantitative

12 functionalization (Table 2). Theoretical molar weight of each functionalized polymer (2a-2f) was

13 calculated considering the average degree of polymerization obtained in the previous MALDI-TOF

14 MS analysis (see Table 1) and the 2-bromo-2-methylpropanoate moiety.

15 Table 2. Esterification of poly(glycol)s with 2-bromo-2-methylpropanoyl bromide in $\mathrm{CH}_{2} \mathrm{Cl}_{2}$

\begin{tabular}{ccccc}
\hline Entry & Yield (\%) $^{\text {Functionalization } \text { degree }^{\mathbf{a}}}$ & $\begin{array}{c}\mathbf{F}_{\mathbf{n}}(\mathbf{( D )}) \\
\mathbf{( \% )}\end{array}$ & DP $_{\text {MALDI }}$ \\
\hline $\mathbf{2 a}$ & 93 & 97 & 2143.747 & 41.49 \\
$\mathbf{2 b}$ & 94 & 94 & 3685.029 & 76.48 \\
$\mathbf{2 c}$ & 92 & 93 & 6192.879 & 133.41 \\
$\mathbf{2 d}$ & 97 & 98 & 873.938 & 15.73 \\
$\mathbf{2 e}$ & 95 & 95 & 2107.907 & 43.74 \\
$\mathbf{2 f}$ & 93 & 98 & 5279.723 & 115.74 \\
\hline
\end{tabular}

${ }^{\mathrm{a}}$ Functionalization degree calculated from ${ }^{1} \mathrm{H}$ NMR spectrum.

$17{ }^{\mathrm{b}}$ Theoretical $M_{\mathrm{n}}$ as calculated from the average DP from MALDI-TOF MS analysis. 
2 Molar mass distribution analysis. The main purpose for the estimation of the molar mass 3 distributions of PEG or MPEG esters of 2-bromo-2-methylpropanoate (2a-f) was to ensure that 4 esterification was the sole reaction that had taken place during the preparation of these materials. The 5 chromatographic separations of all polymers were performed by SEC. The results obtained shown 6 that dispersities remain in the same values $(\nexists<1.11)$ and slight increments in their molecular weights

7 (see Table S-1 in Supporting Information) typical chromatograms are depicted in Figure 3. These 8 curves indicate monomodal molar mass distributions and low molar mass dispersities. Nevertheless, 9 one could not exclude any dimerization or other side reaction under uncontrolled conditions. This 10 concern was based upon the possible reaction between the bromine atom at the methylpropanoate 11 group with trimethylamine, which was present in excess during the esterification reaction. This base 12 might give a hydro-bromo elimination reaction leading to the formation of methacrylate functional 13 group that could eventually undergo a dimerization reaction [29].

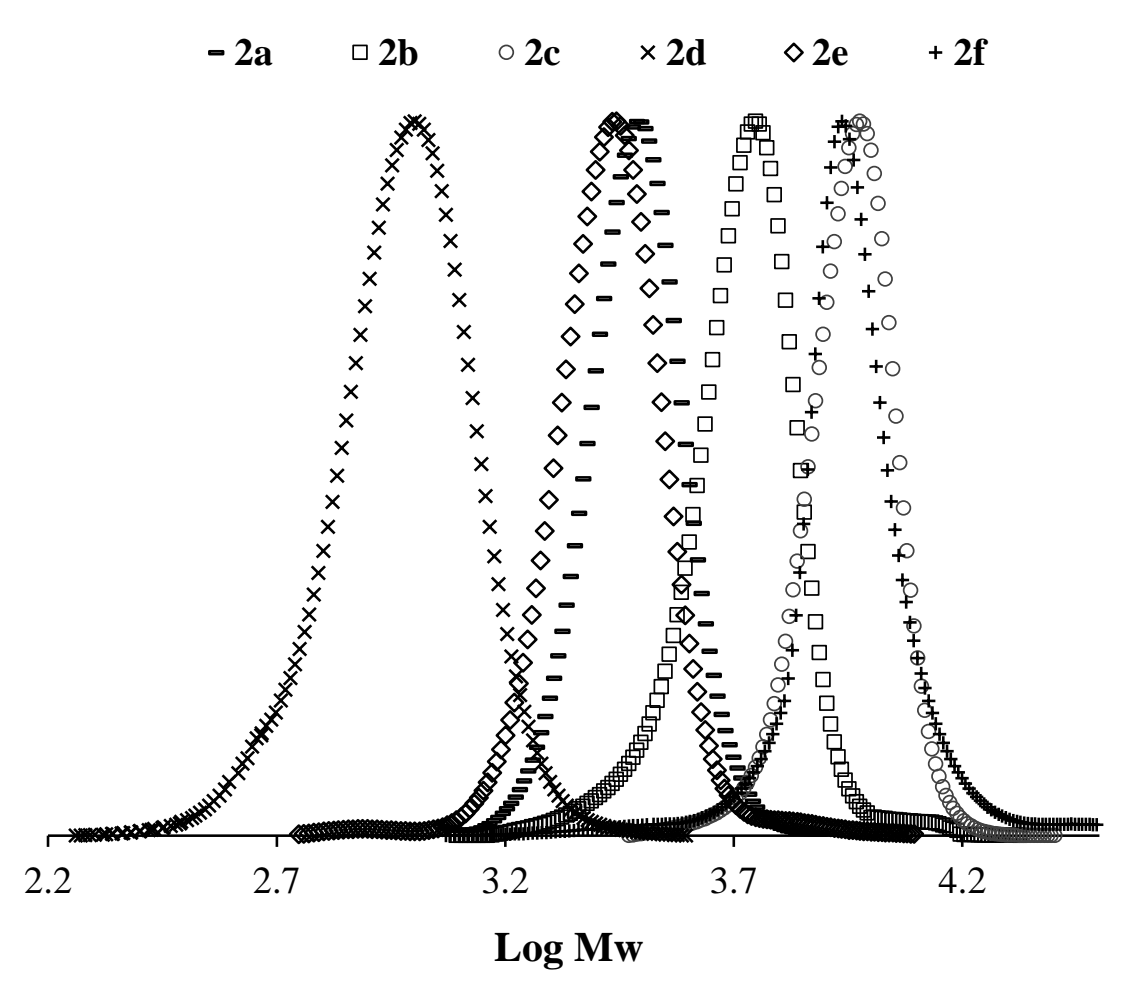

14

Figure 3. Molar mass distribution of esters of 2-bromo-2-methylpropanoate of poly(glycol)s (2a-f).

MALDI-TOF MS investigation of bromine end-groups. End-functionalized polymers with 17 halogen groups can be used as macroinitiators for the preparation of complex macromolecular architectures. However, these halogenated end groups must be characterized by a proper technique. 
1 Focusing on polymers obtained by ATRP, unsaturated groups at the chain end are commonly

2 observed in MALDI-TOF mass spectra. This apparently originates through the loss of the halogen

3 atom $(\mathrm{X}=\mathrm{Br}, \mathrm{Cl})$ during the MALDI-TOF MS analysis [29]. Formation of the unsaturated

4 compounds may also occur as a result of lack of control during the ATRP polymerization process,

5 due to the free-radical disproportionation at the growing chain end [34]. Thus, the problem is to

6 discriminate between these two alternatives in order to establish an accurate structure of ATRP

7 macroinitiators. As previously discussed, end-functionalized esters of PEG or MPEG with 2-bromo-

8 2-methylpropanoate were prepared via esterification. With this strategy, the presence of unsaturated

9 polymers was avoided, because poly(glycol)s with narrow dispersities as starting reagent were used.

10 In the literature, in some protocols of analysis for PEG or MPEG by MALDI-TOF-MS avoid the use

11 of cationic agents, in spite of this species like $[\mathrm{M}+\mathrm{H}]^{+},[\mathrm{M}+\mathrm{Na}]^{+}$and $[\mathrm{M}+\mathrm{K}]^{+}$have been found, this

12 fact could be explained considering that the protonated species came from the matrix and the alkaline

13 species from the residues of these alkaline metals present in the water or solvent used [35][36]. For

14 this reason, our first approach, was centered in the analysis of the polymers 2a and 2e by MALDI-

15 TOF MS with DCTB matrix without any cationic agent. Poor ion generation was found in this

16 analysis without the cationization agent and not sufficient for a precise analysis of the halogen end

17 functionalization of ATRP macroinitiators. These results were compared with the samples in which

18 AgTFA was used as the cationic agent and clear differences could be seen in the ion formation, these

19 spectra were included in the supplemental information (Figures $S$-2 and $S$-3).

20 Taking into account the previous results, it was evaluated the influence of the cationizing agents with

21 different acid strengths, like silver trifluoromethanesulfonate (AgTFS) or silver trifluoroacetate

22 (AgTFA), and with absolute hardness value like sodium trifluoroacetate (NaTFA). Their nature was

23 evaluated in the ionization process, as well as their effect in the occurrence of secondary peaks in the

24 MALDI-TOF MS spectra. Under these conditions, it was observed a higher signal intensity,

25 indicating good ionization in each case. In all the evaluated samples, ion sequences were separated

26 by a nominal value close to $44.053 \mathrm{Da}$, which was consistent with the molar mass of the repeating

27 unit $\left(\mathrm{OCH}_{2} \mathrm{CH}_{2}\right)$ present in the glycol polymers. Different molecular species were observed

28 depending on the cationization salt or solvent employed. The detailed discussion of this results is

29 given in the following sections.

30 Influence of ionic agents during MALDI-TOF MS analysis. For this MALDI-TOF-MS study,

31 DCTB was employed as the matrix, silver trifluoroacetate as a cationic agent and methanol as the

32 solvent. As stated before, the mechanism for the interactions between the silver and the polymeric 
1 analyte remains unknown [37]. However, it was proposed that the reaction between silver salts with

2 halogenated compounds occurred prior to the excitation, in other words, before the impact of the laser

3 source into the matrix. For example, Tintaru et al. [27] concluded that the elimination of $\mathrm{HBr}$ had

4 most likely ocurred during sample preparation.

5 In this study, a molar ratio for DCTB/polymer/AgTFA of 310/1/18 was used. An efficient ionization

6 was thus obtained for the six esters of 2-bromo-2-methylpropanoate of PEG or MPEG (2a-f). The

7 analysis was focused on the peaks with the highest intensity, the experimental $\mathrm{m} / \mathrm{z}$ ratio was compared

8 to the calculated molar mass of the possible structures. The molar mass distribution of the ester $\mathbf{2 a}$

$9(D P=41)$ obtained in the MALDI analysis is shown in Figure 4. In this Figure, two patterns were

10 clearly identified, that with the highest intensity was assigned to the unsaturated termination (structure

11 as shown in Figure 5a and named as U Series).

12

13

14

15
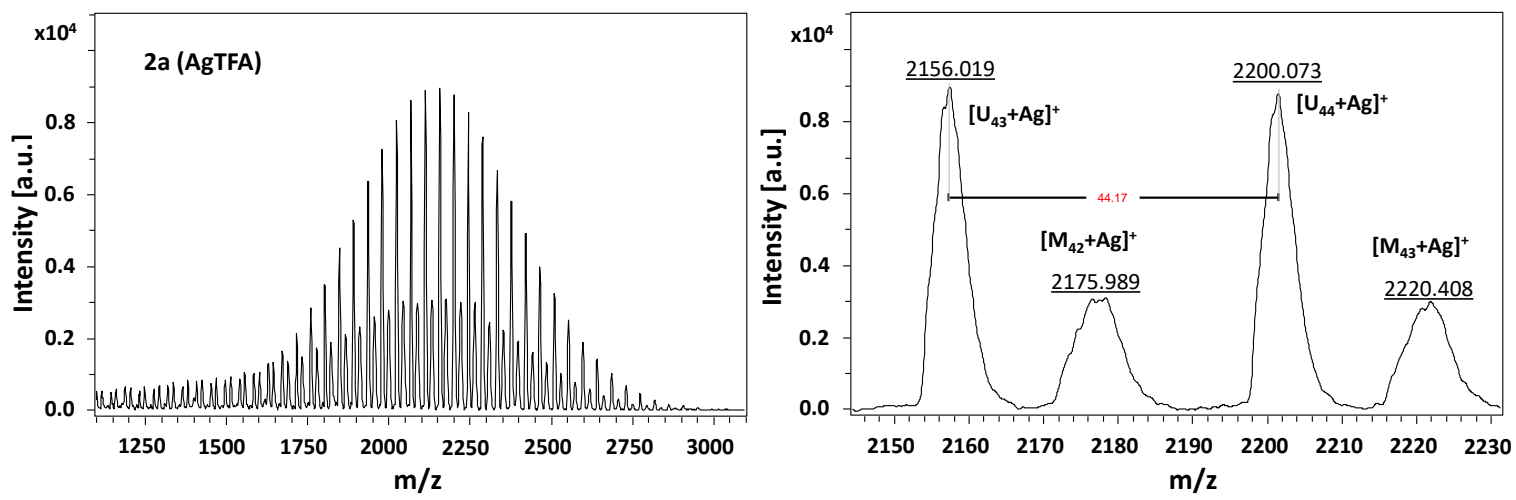

Figure 4. Molar mass distribution of PEG bis(2-bromo-2-methylpropanoate) $D P=41$ (2a) obtained by MALDI in reflectron mode with AgTFA as cationic agent.

The unsaturated compound was formed due to the elimination of bromine from the original ester $\mathbf{2 a}$. The explanation for this observation arises from the fact that silver $\left(\mathrm{Ag}^{+}\right)$has a low cationic absolute hardness value $(\eta=6.9 \mathrm{eV})$, and this cation has the ability to react with halo-organic compounds with a softer character. However, it was reported that, as a result of this interaction, a nucleophilic substitution was promoted [36]. Through this reaction, the loss of the terminal bromine could be promoted by a silver cation giving a complex, this intermediate reacting with the conjugated acid of the cationic agent and thus giving an unsaturated compound, a silver bromide and the corresponding acid. The second ion sequence was assigned to methoxylated products formed during the interaction of the intermediate and methanol that was used as a solvent. These methoxylated derivatives can be observed in Figure 4 as secondary peaks with the lowest intensity (structure as depicted in Figure $\mathbf{5 b}$ and named as M Series). The proposed mechanism is displayed in Scheme 2. 
a) U Series

b) M Series

c) W Series<smiles>C=C(C)C(=O)OCCC(C)OC(=O)C(=C)C</smiles><smiles>CC[14CH2][14CH2]C(C)(C)C(=O)OCCOC(=O)C(C)(C)O</smiles>

Figure 5. Compounds identified in MALDI analysis of bis(2-bromo-2-methylpropanoate) of PEG.<smiles></smiles>

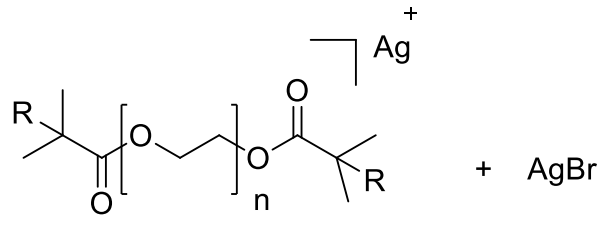

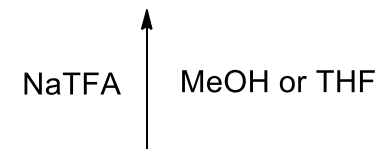
Nucleophilic
Substitution<smiles>CC(OCCOC(=O)C(C)(C)Br)C(=O)C(C)(C)Br</smiles>

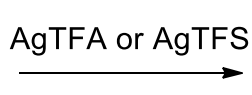<smiles>COC(=O)C(C)(C)OCCOC(=O)C(C)(C)Br</smiles>

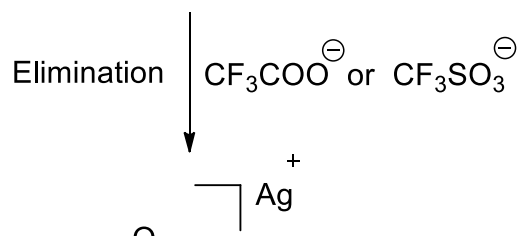

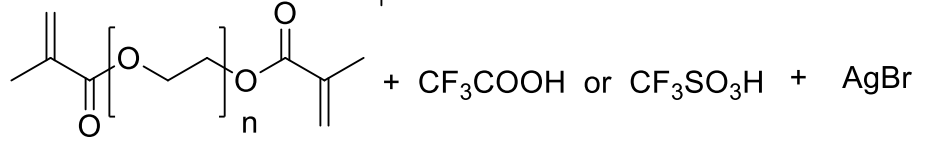

5 Scheme 2. Proposed mechanisms for 2-bromo-2-methylpropanoato end-functionalized esters during 6 MALDI-TOF analysis with $\mathrm{Ag}^{+}$and $\mathrm{Na}^{+}$as cationic agents.

8 With an increase in molar mass of the esters derived from PEG, a reversal in the sequence intensities 9 was observed (entries $\mathbf{2 b}$ and $\mathbf{2 c}$ in Table 3). In these samples, the methoxylated esters had greater 10 intensities than those of the unsaturated compounds, when employing the same ionization system (see 11 Figure S-4 and Figure S-5 in Supporting Information). In the esterified MPEG a similar behavior was 12 found, unsaturated end groups were identified as the main products (entries 2d-e in Table 3). 
1 Nonetheless, a secondary ion sequence pattern with lower intensity was identified arising from

2 hydroxyl substitution of the brominated esters (W Series in Figure 5c). In the case of a higher molar

3 mass sample (2f), the W Series was higher in intensity than the U Series (entry 2f, Table 3). It was

4 clear that with the increase in molar mass, a decrease in the unsaturated products was observed. The

5 MALDI-TOF mass spectra of MPEG esters are illustrated in Supporting Information (Figures S-6 to

$6 S$-8). In all experiments with AgTFA/methanol, it was clear that with the increment of the molar mass

7 in PEG or MPEG esters, a decrement in the unsaturated products was observed.

8

9 Table 3. Results for MALDI-TOF MS analysis of brominated esters 2a-f with $\mathrm{DCTB} / \mathrm{AgCF}_{3} \mathrm{COO}$ 10 and $\mathrm{MeOH}$ as the solvent.

\begin{tabular}{|c|c|c|c|c|c|c|c|c|}
\hline \multirow[b]{2}{*}{ MI } & \multirow[b]{2}{*}{$D P_{p^{a}}$} & \multirow[b]{2}{*}{$\begin{array}{c}M_{\mathbf{p}} \mathbf{M I}^{\mathrm{b}} \\
\left(\left[\mathbf{M}_{\mathbf{p}}+\mathbf{A g}\right]^{+}\right) \\
(\mathbf{D a})\end{array}$} & \multicolumn{3}{|c|}{ Peaks with Highest Intensity } & \multicolumn{3}{|c|}{ Peaks with Lowest Intensity } \\
\hline & & & $\begin{array}{c}M_{\mathrm{p}} \text { found } \\
\left(\left[\mathrm{M}_{\mathrm{p}}+\mathbf{A g}\right]^{+}\right) \\
(\mathrm{Da})\end{array}$ & $\begin{array}{l}\text { Assigned } \\
\text { Structure }\end{array}$ & $\begin{array}{c}M_{\mathrm{p}} \\
\text { Calculated } \\
\left(\left[\mathrm{M}_{\mathrm{p}}+\mathrm{Ag}\right]^{+}\right) \\
(\mathrm{Da}) \\
\end{array}$ & $\begin{array}{c}M_{\mathrm{p}} \text { found } \\
\left(\left[\mathrm{M}_{\mathrm{p}}+\mathrm{Ag}\right]^{+}\right) \\
(\mathrm{Da})\end{array}$ & $\begin{array}{l}\text { Assigned } \\
\text { Structure }\end{array}$ & $\begin{array}{c}M_{\mathrm{p}} \\
\text { calculated } \\
\left(\left[\mathrm{M}_{\mathrm{p}}+\mathbf{A g}\right]^{+}\right) \\
(\mathbf{D a}) \\
\end{array}$ \\
\hline $\mathbf{2 a}$ & 43 & 2341.125 & 2156.019 & {$[\mathrm{U}+\mathrm{Ag}]^{+}$} & 2156.332 & 2220.408 & {$[\mathrm{M}+\mathrm{Ag}]^{+}$} & 2220.396 \\
\hline $2 \mathbf{b}$ & 74 & 3706.768 & 3585.934 & {$[\mathrm{M}+\mathrm{Ag}]^{+}$} & 3586.039 & 3521.792 & {$[\mathrm{U}+\mathrm{Ag}]^{+}$} & 3521.989 \\
\hline $2 c$ & 131 & 6217.789 & 5988.232 & {$[\mathrm{M}+\mathrm{Ag}]^{+}$} & 5989.192 & 5924.564 & {$[\mathrm{U}]^{+}$} & 5925.167 \\
\hline 2d & 15 & 972.682 & 869.487 & {$[\mathrm{U}+\mathrm{Ag}]^{+}$} & 868.787 & 887.530 & {$[\mathrm{~W}+\mathrm{Ag}]^{+}$} & 886.795 \\
\hline $2 e$ & 44 & 2250.219 & 2146.178 & {$[\mathrm{U}+\mathrm{Ag}]^{+}$} & 2146.337 & 2164.480 & {$[\mathrm{~W}+\mathrm{Ag}]^{+}$} & 2164.332 \\
\hline $2 f$ & 117 & 5466.088 & 5380.624 & {$[\mathrm{~W}+\mathrm{Ag}]^{+}$} & 5380.201 & 5362.534 & {$[\mathrm{U}+\mathrm{Ag}]^{+}$} & 5362.238 \\
\hline
\end{tabular}

11 a $D P$ value corresponding to $M_{\mathrm{p}}$.

$12{ }^{\mathrm{b}}$ Theoretical molar mass of macroinitiators (MI) related to corresponding $D P_{\mathrm{p}}$ value.

13 Tintaru et al. [27] also reported the presence of unsaturated and methoxylated species obtained from

14 the loss of the halide end group in bromine end-functionalized $\omega$-polystyrene samples analyzed by

15 MALDI TOF MS with silver cationic agents. They concluded that the elimination reaction took place

16 during the sample preparation, prior to the ionization; this assumption was based on NMR analysis

17 of the samples.

18 Here, the reaction between MPEG 2-bromo-2-methylpropanoate ester (2d) with silver trifluoroacetate 19 (AgTFA) at room temperature (298 K) was studied by NMR employing deuterated methanol as 20 solvent. In this analysis, only the reaction of the ester with the silver salt was considered with molar 21 ratio of 1:5 MPEG 2-methyl-2-bromopropionate (2d): AgTFA, without the use of DCTB. Bearing in 22 mind that the main function of this matrix in the MALDI analysis was to transfer the energy from the 
1 laser irradiation to the sample during the ionization process, it did not have any influence on the

2 bromine elimination reaction. In the ${ }^{1} \mathrm{H}$ NMR spectrum, the chemical shifts of the $\mathbf{2} \mathbf{d}$ ester could be

3 appreciated (see Figure S-9 in Supporting Information). This ester was stable for at least 60 s; during

4 this time, there were no observable signals in the ${ }^{1} \mathrm{H}$ NMR spectrum for the unsaturated compound

5 (2dU). After this time lapse, a very low yield $(<6 \%)$ of the unsaturated product (U Series) was

6 identified; its concentration was calculated by the integration values for the signals of the vinyl

7 protons at 6.13 and $5.66 \mathrm{ppm}$ respectively and compared to the signals for the $\mathrm{CH}_{2}$ protons from the

8 carbonyl ester at $4.27 \mathrm{ppm}$.

9 The methoxylated or hydroxylated added products (M or W Series) at the end of the polymer chains

10 were characterized indirectly by changes in the magnetic environment surrounding the 2-methyl

11 group of the 2-methylpropanoate moiety. The methyl group near the bromine atom had a chemical

12 shift of $1.95 \mathrm{ppm}$ in the ester $\mathbf{2 d}$. It shifted to $2.06 \mathrm{ppm}$ in the unsaturated product (U Series) and to

$131.20 \mathrm{ppm}$ in the hydroxylated or methoxylated ester (M Series). The evolution of these two products

14 was followed and quantified during $700 \mathrm{~s}$ at $298 \mathrm{~K}$, the concentration of these two products remaining

15 constant with minimal variation during this time.

16 In order to emulate the ionization process, the temperature of the NMR probe was increased [38] from

17 room temperature (298K) to $313 \mathrm{~K}$, and changes in the mixture of $\mathbf{2 d}$ and AgTFA in deuterated

18 methanol were monitored, the results are depicted in Figure 6. The concentration of the M Series

19 increased to $50 \%$ after $300 \mathrm{~s}$, while the concentration of the unsaturated compound (U Series)

20 remained constant throughout the entire experiment. According to these observations, we could

21 conclude that the changes in the structure of the brominated esters would be promoted by an energy

22 source such as the ionization process and not during sample preparation. The increase in the

23 concentration of substitution products ( $M$ Series) at higher temperature instead of that of the

24 elimination products (U Series) suggested the presence of an intermediate promoted by the reaction between silver ion and the bromine atom. 


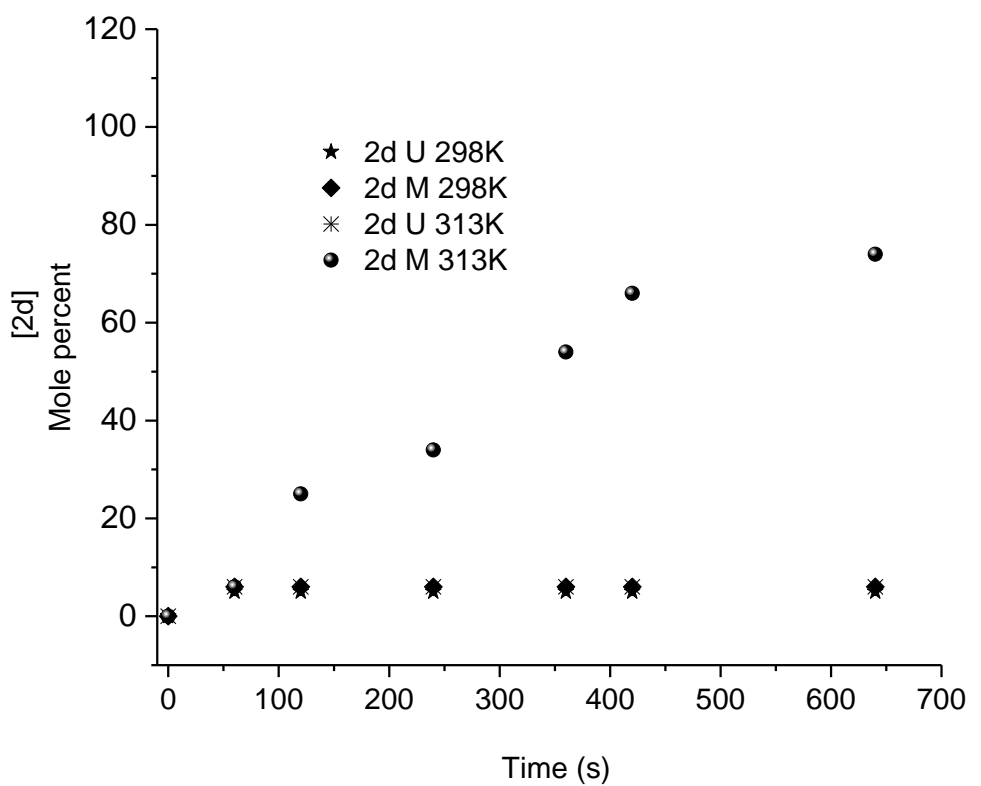

1

2 Figure 6. Influence of AgTFA on stability of the $\mathbf{2 d}$ ester obtained by ${ }^{1} \mathrm{H}$ NMR spectroscopy in 3 deuterated methanol.

4 In order to explain the previous results and how the end groups are formed during the MALDI 5 ionization process, the possibility of $\gamma$-hydrogen abstraction of PEG or MPEG esters followed by the 6 formation of an intermediate and a subsequent substitution or elimination reaction promoted by the 7 interaction with the silver ion was considered. It was then proposed the use of silver 8 trifluoromethanesulfonate $\left(\mathrm{AgCF}_{3} \mathrm{SO}_{3}\right)$ as a cationic agent in which the conjugated acid of the 9 counterion has a different acid strength and would influence the presence of the elimination 10 compounds. MALDI conditions with an equal molar ratio of $\mathrm{DCTB} / \mathbf{2 a - f} / \mathrm{AgCF}_{3} \mathrm{SO}_{3}$ of 310/1/18 were 11 selected for the new series of experiments. Excellent ionization of the six 2-bromo-212 methylpropanoate esters derived from PEG or MPEG was found. In Figure 7, the MALDI TOF MS 13 spectra for the 2a ester are shown. Two patterns were mainly observed, i.e. the $\mathrm{U}$ and $\mathrm{M}$ Series, which 14 corresponded to the elimination or substitution products. The mass spectra of the other macroinitiators 15 (2b-f) are shown in Supporting Information (Figure S-9 to Figure S-13). 

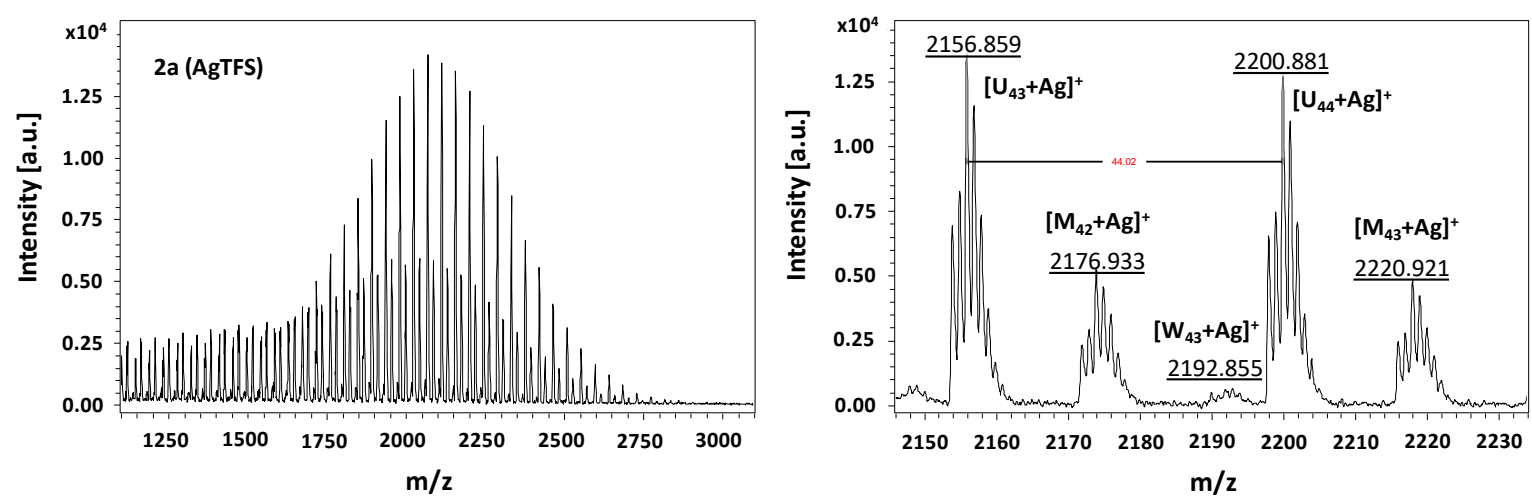

1

Figure 7. MALDI-TOF spectra of the $\mathbf{2 a}$ brominated ester using AgTFS/2a/DCTB with a molar ratio of 310/1/18 employing methanol as the solvent, obtained in reflectron mode.

The $m / z$ ratios obtained in each MALDI-TOF mass spectrum were compared with the theoretical molar masses of the possible structures, and the results are listed in Table 4. When the six brominated esters were compared with the esters characterized with AgTFA as the ionic agent, a similar behavior was observed under the new ionization conditions. The loss of the bromine atom was also observed, thus contributing to elimination or substitution reactions. The main products corresponded to $\mathrm{U}, \mathrm{M}$ or W Series, respectively. In the macroinitiators with molar masses lower than or equal to 2,000 Da, the products from elimination were preponderant (U Series), and with the increment of the molar mass, the formation of addition products was observed (M Series).

Table 4. Results for MALDI-TOF MS analysis of brominated esters 2a-f with DCTB/AgCF $\mathrm{SO}_{3}$ and $\mathrm{MeOH}$ as the solvent.

\begin{tabular}{|c|c|c|c|c|c|c|c|c|}
\hline \multirow[b]{2}{*}{$\begin{array}{c}\mathbf{M} \\
\mathbf{I}\end{array}$} & \multirow[b]{2}{*}{$\underset{\mathbf{a}}{D P_{p}}$} & \multirow[b]{2}{*}{$\begin{array}{c}M_{\mathrm{p}} \text { PEG- } \\
\mathrm{Br}^{\mathrm{b}} \\
\left([\mathrm{Mp}+\mathrm{Ag}]^{+}\right. \\
)(\mathrm{Da})\end{array}$} & \multicolumn{3}{|c|}{ Peaks with Highest Intensity } & \multicolumn{3}{|c|}{ Peaks with Lowest Intensity } \\
\hline & & & $\begin{array}{l}M_{\mathrm{p}} \text { found } \\
([\mathrm{Mp}+\mathrm{Ag}] \\
\quad+(\mathrm{Da})\end{array}$ & $\begin{array}{c}\text { Assigned } \\
\text { Structur } \\
\text { e }\end{array}$ & $\begin{array}{c}M_{\mathbf{p}} \\
\text { Calculate } \\
\mathbf{d} \\
\begin{array}{c}{[\mathrm{Mp}+\mathbf{A g}]} \\
+(\mathrm{Da})\end{array}\end{array}$ & $\begin{array}{c}M_{\mathrm{p}} \text { found } \\
\left([\mathrm{Mp}+\mathrm{Ag}]^{+}\right. \\
\quad)(\mathrm{Da})\end{array}$ & $\begin{array}{c}\text { Assigned } \\
\text { Structur } \\
\text { e }\end{array}$ & $\begin{array}{c}M_{\mathbf{p}} \\
\text { calculated } \\
{ }_{\left([\mathrm{Mp}+\mathbf{A g}]^{+}\right.} \\
)(\mathrm{Da})\end{array}$ \\
\hline $\mathbf{2 a}$ & 43 & 2341.125 & 2156.859 & {$[\mathrm{U}+\mathrm{Ag}]^{+}$} & 2156.332 & 2220.921 & {$[\mathrm{M}+\mathrm{Ag}]^{+}$} & 2220.396 \\
\hline $2 \mathrm{~b}$ & 74 & 3706.768 & 3585.627 & {$[\mathrm{M}+\mathrm{Ag}]^{+}$} & 3586.039 & 3558.687 & {$[\mathrm{~W}+\mathrm{Ag}]^{+}$} & 3557.985 \\
\hline 2c & 131 & 6217.789 & 6070.653 & {$[\mathrm{~W}+\mathrm{Ag}]^{+}$} & 6069.006 & 6096.611 & {$[\mathrm{U}+\mathrm{Ag}]^{+}$} & 6097.060 \\
\hline 2d & 15 & 972.682 & 869.089 & {$[\mathrm{U}+\mathrm{Ag}]^{+}$} & 868.787 & 887.093 & {$[\mathrm{~W}+\mathrm{Ag}]^{+}$} & 886.795 \\
\hline $2 e$ & 44 & 2250.219 & 2147.411 & {$[\mathrm{U}+\mathrm{Ag}]^{+}$} & 2146.337 & 2165.427 & {$[\mathrm{~W}+\mathrm{Ag}]^{+}$} & 2164.332 \\
\hline $2 \mathbf{f}$ & 117 & 5466.088 & 5380.217 & {$[\mathrm{~W}+\mathrm{Ag}]^{+}$} & 5380.201 & 5362.621 & {$[\mathrm{M}+\mathrm{Ag}]^{+}$} & 5362.238 \\
\hline
\end{tabular}


${ }^{\text {a }} D P$ value corresponding to $M_{\mathrm{p}}$

${ }^{\mathrm{b}}$ Theoretical molar mass related to corresponding $D P_{\mathrm{p}}$ value

As it could be seen in Table 3, the ions with the highest intensity displayed the same tendency as the previous results with the presence of unsaturated compounds in esters with molar masses lower than or equal to 2,000 Da. Nevertheless, for the esters with molar masses higher than 2,000 Da (2b, 2cc, and $2 \mathbf{f}$ samples), no unsaturated end groups were detected. With these results, it could be mentioned that the increment of the acidity of the counterion in the cationic agent would not have an influence on the MALDI-TOF MS spectra. It is noteworthy that the esters, the matrix (DCTB), and the cationization agent were dissolved in methanol, then a small aliquot of the mixture was deposited to a sample plate and the matrix and polymer co-crystallized as the solvent evaporated. During this process, methanol or water molecules might be trapped in the formed crystals, depending on the environment conditions. At this stage, the polymers were arranged in the crystal according to their polarities. Thus, functionalized polymers with lower molar masses had the 2-metyl-2bromopropionate end group available to interact with the cationic agent, followed by the proton abstraction to produce unsaturated polymer. On the contrary, in polymers with molar masses higher than 2,000 Da, the end group would be distant from the cationic agent, thus it was easier to react with the rest of the solvent/water molecules, leading to the substitution products in the M or W Series observed in the mass spectra.

Considering the previous results, when specific ionization conditions were employed, the bromine atom was not present in the ATRP macroinitiators, due to its lability. This inaccurate analysis may cause an uncertainty in the quality of the ATRP macroinitiators. In this study, the silver ion reacts with the $\alpha$-bromo carbonyl compound, due to their similar soft characteristic. Metals with higher absolute hardness value, such as sodium $(\eta=21.1 \mathrm{eV})$, must have different reactivity with the halocarbonyl compound and will be more stable during the laser excitation in the ionization process on the MALDI analysis.

With the purpose to prove the low reactivity of the $\alpha$-halo-carbonyl compound with alkaline metals, sodium trifluoroacetate (NaTFA) was selected as a cationic agent. All the samples were analyzed with the same conditions and reagents as used before, DCTB as the matrix in methanol and a molar ratio of DCTB/brominated esters 2a-f/NaTFA of 310/1/18. The MALDI-TOF mass spectra of all the esters revealed the presence of a single pattern of ions. Therefore, it may be deduced some stability in the molecular ions during the ionization process. This result confirmed the fact that the sodium cation had a low reactivity with the $\alpha$-bromo esters, and no elimination or addition products (U, M 
1 or W Series respectively) were identified in the mass spectra. In contrast, some secondary peaks with

2 very low intensity were identified as molecular ions without the presence of cationic agent $\left(\mathrm{Na}^{+}\right)$.

3 As a typical example, Figure 8 shows the molar mass distribution for the 2a sample, in which two 4 molecular species were identified as PEG molecules with 2-methyl-2-bromopropionate with and 5 without counterion. These molecular structures (B Series in Figure 10a) were assigned based on the $6 \mathrm{~m} / \mathrm{z}$ ratio of ions observed in each mass spectrum and compared with the calculated molar mass for 7 the brominated esters (2a-f). In all cases, the experimental isotopic pattern fitted well with the 8 theoretical one, as it can be seen in Table 5. The mass spectra for $\mathbf{2 b}$ and $\mathbf{2 c}$ samples are shown in $9 \quad$ Figure S-14 and Figure S-15 in Supporting Information. It is important to mention that these samples 10 (2a-2c) are dibromated esters, thus the resulting isotopic pattern in all mass spectra is due to natural 11 bromine isotopes $\left({ }^{79} \mathrm{Br}\right.$ and $\left.{ }^{81} \mathrm{Br}\right)$.
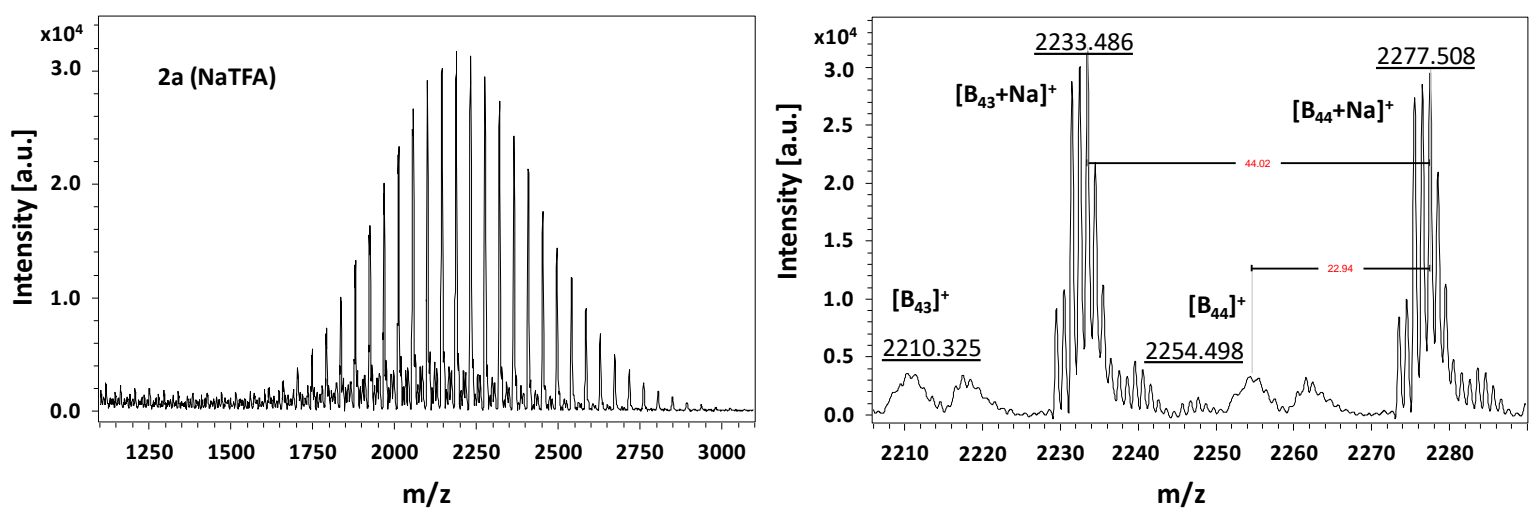

12

Figure 8. MALDI-TOF spectra of the brominated ester 2a using NaTFA/2a/DCTB with a molar ratio of 310/1/18 employing methanol as the solvent, obtained in reflectron mode.

The MALDI-TOF mass spectra of the MPEG 2-methyl-2-bromopropionate esters $\mathbf{2 d - f}$ also displays the bromine isotopic peaks as the main ions in the molar mass distribution curve. The mass spectrum for the 2e sample is shown in Figure 9. The MPEG molecules with bromine end-functionalization were identified as BM Series (see structure in Figure 10b). These MALDI-TOF results confirmed that the stabilization of the macroinitiators was achieved in the presence of the sodium cation. This high stability was sufficient to detect the molecular ions of the esters $\mathbf{2 d - f}$, where the cationic agent is absent $\left(\mathrm{BM}^{+}\right.$Series in Figure 9). The results for $\mathbf{2 d}$ and $\mathbf{2 f}$ samples are shown in Figure S-16 and Figure S-17 in Supporting Information. 
1

Table 5. Results for MALDI-TOF-MS analysis of brominated esters 2a-f in DCTB/NaTFA and $13 \mathrm{MeOH}$ as the solvent.

Figure 9. MALDI-TOF spectra of the brominated ester 2e using NaTFA/2e/DCTB with a molar ratio of 310/1/18 employing methanol as the solvent, obtained in reflectron mode. a) B Series<smiles></smiles>

b) BM Series<smiles>CCCCCOC(=O)C(C)(C)Br</smiles>

Figure 10. Compounds identified in MALDI analysis of bis(2-bromo-2-methylpropanoate) of PEG and MPEG with NaTFA/DCTB in methanol.

Further, the results listed in Table 5 showed that the molar mass of the PEG 2-methyl-2bromopropionate esters 2a-c or MPEG 2-methyl-2-bromopropionate esters $\mathbf{2 d - f}$ had no influence on the ionization and detection process in MALDI-TOF analysis. In all cases, the molar mass of the brominated or dibrominated esters were accurately determined and the high quality of the ATRPmacroinitiators was established.

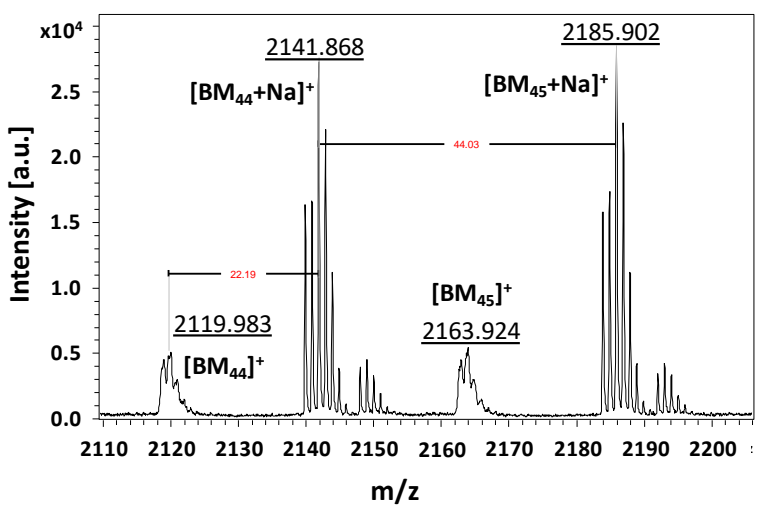

\begin{tabular}{|c|c|c|c|c|c|c|c|c|}
\hline \multirow[b]{2}{*}{$\begin{array}{c}\text { M } \\
\text { I }\end{array}$} & \multirow[b]{2}{*}{$\underset{\mathrm{a}}{\boldsymbol{D} \boldsymbol{P}_{\mathbf{p}}}$} & \multirow[b]{2}{*}{$\begin{array}{c}M_{\mathrm{p}} \text { PEG- } \\
\mathrm{Br}^{\mathrm{b}} \\
([\mathrm{Mp}+\mathrm{Na}] \\
+(\mathrm{Da})\end{array}$} & \multicolumn{3}{|c|}{ Peaks with Highest Intensity } & \multicolumn{3}{|c|}{ Peaks with Lowest Intensity } \\
\hline & & & $\begin{array}{l}M_{\mathrm{p}} \text { found } \\
([\mathrm{Mp}+\mathrm{Na}] \\
\quad+(\mathrm{Da})\end{array}$ & $\begin{array}{c}\text { Assigned } \\
\text { Structur } \\
\text { e }\end{array}$ & $\begin{array}{c}M_{\mathrm{p}} \\
\text { calculated } \\
([\mathrm{Mp}+\mathrm{Na}]+ \\
)(\mathrm{Da})\end{array}$ & $\begin{array}{l}M_{\mathrm{p}} \text { found } \\
([\mathrm{Mp}+\mathrm{Na}] \\
+(\mathrm{Da})\end{array}$ & $\begin{array}{c}\text { Assigned } \\
\text { Structur } \\
\text { e }\end{array}$ & $\begin{array}{c}M_{\mathrm{p}} \\
\text { calculated } \\
([\mathrm{Mp}+\mathrm{Na}] \\
+(\mathrm{Da})\end{array}$ \\
\hline $\mathbf{2 a}$ & 43 & 2233.257 & 2233.486 & {$[\mathrm{~B}+\mathrm{Na}]^{+}$} & 2233.257 & 2210.325 & {$[\mathrm{~B}]^{+}$} & 2210.267 \\
\hline $2 \mathbf{b}$ & 74 & 3598.900 & 3597.883 & {$[\mathrm{~B}+\mathrm{Na}]^{+}$} & 3598.900 & 3582.040 & {$[\mathrm{~W}]^{+}$} & 3582.276 \\
\hline 2c & 131 & 6109.921 & 6108.93 & {$[\mathrm{~B}+\mathrm{Na}]^{+}$} & 6109.921 & 6085.570 & {$[\mathrm{~B}]^{+}$} & 6086.931 \\
\hline
\end{tabular}




\begin{tabular}{rrr|rcr|rrr}
$\mathbf{2 d}$ & 15 & 864.814 & 865.128 & {$[\mathrm{~B}+\mathrm{Na}]^{+}$} & 864.814 & 843.555 & {$[\mathrm{~B}]^{+}$} & 843.555 \\
$\mathbf{2 e}$ & 44 & 2142.351 & 2141.868 & {$[\mathrm{~B}+\mathrm{Na}]^{+}$} & 2142.351 & 2119.983 & {$[\mathrm{~B}]^{+}$} & 2119.361 \\
$\mathbf{2 f}$ & 117 & 5358.220 & 5295.739 & {$[\mathrm{~W}+\mathrm{Na}]^{+}$} & 5295.323 & 5358.219 & {$[\mathrm{~B}+\mathrm{Na}]^{+}$} & 5358.220 \\
\hline
\end{tabular}

$1 \quad{ }^{a} D P$ value corresponding to $M_{\mathrm{p}}$

$2 \quad{ }^{\mathrm{b}}$ Theoretical molar mass related to corresponding $D P_{\mathrm{p}}$ value

4 Influence of solvent during MALDI-TOF MS analysis. It is important to consider that in MALDI

5 TOF MS analysis, the sample preparation has a strong influence on the ionization process, and

6 definitely it does on the final chemical structure obtained. In this process, the sample will interact

7 with all components, i.e. matrix, cationic agent, and solvent, and depending on its compatibility with

8 the cationic agent, it will produce different compounds rather than the original molecules.

9 The solvent has an important role as it will promote solvation of the metal ion and make it available

10 for any chemical reaction. In addition, the solvent is also used as a vehicle to apply a homogeneous

11 mixture into the target. The solvent is evaporated and the mixture starts to crystallize, and depending

12 on the polarity and the solvent volatility, the sample and cationic agent are available to interact with

13 the energy source. For this instance, the effect of THF as a common solvent on the characterization

14 of PEG or MPEG 2-methyl-2-bromopropionate esters in the MALDI-TOF MS analysis was

15 evaluated. The six brominated esters 2a-f were analyzed using the same reagents and molar ratios

16 (see Table S-2 to S-4 in Supporting Information).

17 According to the collected results, the use of THF had no influence on the ionization process of the

18 ATRP initiators. Indeed, the same molar mass distribution patterns were obtained when compared to 19 the results with methanol as a solvent. In the latter case, the main structures obtained with the sodium 20 cation were dibrominated molecular ions of the esters $2 \mathbf{a}-\mathbf{f}$ and a second pattern was identified for the 21 molecular ions without the sodium metal, as is evidenced in Figure 11 and Figure 12 for the esters 22 2a and 2e. This study also demonstrated that the cationic metal agent was the main responsible of the 23 formation of unsaturated, methoxylated or hydroxylated esters. 

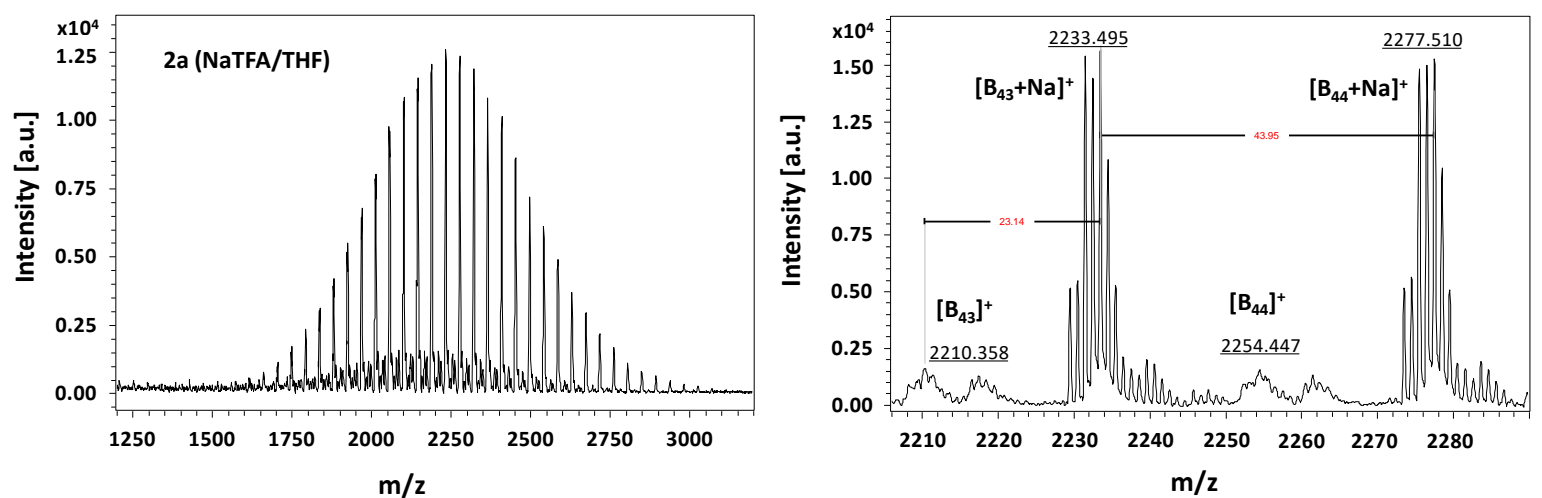

Figure 11. MALDI-TOF spectra of the dibrominated ester 2a using NaTFA/2a/DCTB with a molar ratio of 310/1/18 and THF as the solvent, obtained in reflectron mode.
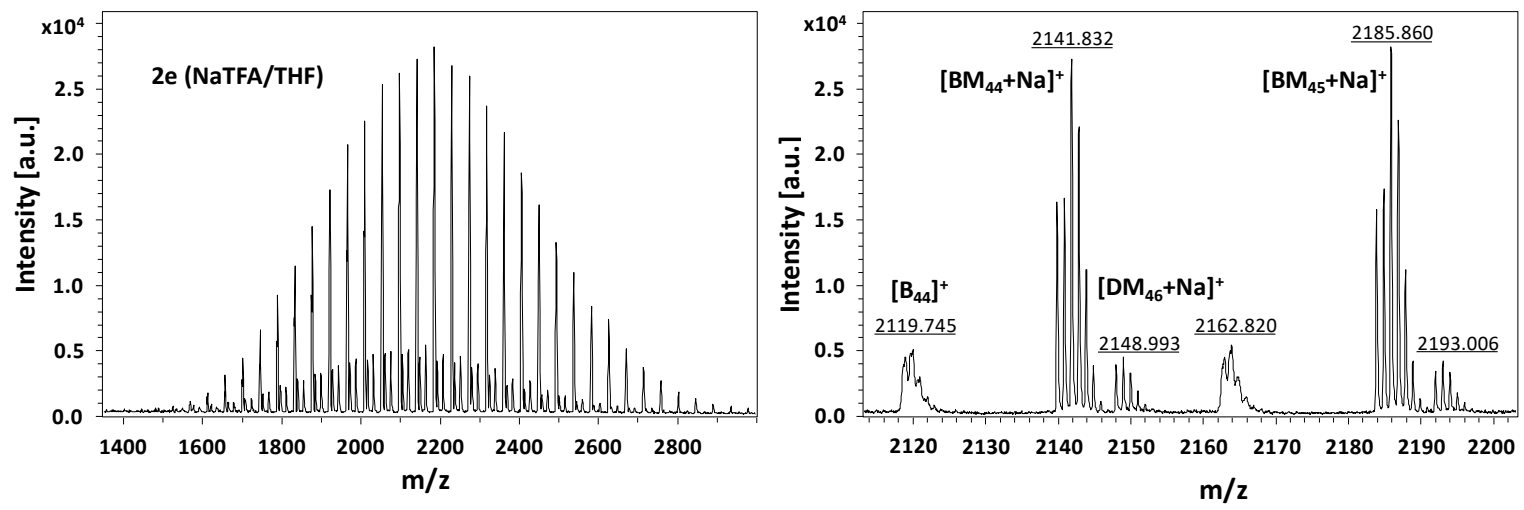

Figure 12. MALDI-TOF spectra of the brominated ester 2e using NaTFA/2e/DCTB with a molar ratio of 310/1/18 and THF as the solvent, obtained in reflectron mode.

\section{Conclusions}

The effect of the cationic agents on the determination of the molar mass by MALDI-TOF MS for PEG or MPEG with 2-bromo-2-methylpropanoate end groups was thoroughly investigated. Two silver salts (trifluoroacetate or trifluoromethanesulfonate) and one sodium salt (trifluoroacetate) in methanol or tetrahydrofuran were chosen as promoters of cationic species and trans-2-[3-(4-tertbutylphenyl)-2-methyl-2-propenylidene] malononitrile (DCTB) as the matrix. It was established that the silver ions reacted with the $\alpha$-bromo carbonyl compound, due to their similar soft characteristic, enhancing the loss of the bromine atom, thus contributing to elimination or substitution products. This reaction was confirmed by ${ }^{1} \mathrm{H}$ NMR experiments, from which it was found that it was triggered when the source of energy was applied. No reaction with the halo-carbonyl compounds was observed 
1 with the use of sodium as cationic agent, thus resulting in more stable molecular ions during the laser

2 excitation in the MALDI analysis. Poly(glycol)s with 2-bromo-2-methylpropanoate end-

3 functionalization could be efficiently used as hydrophilic macroinitiators in ATRP polymerizations,

4 because their structure is accurately characterized by MALDI-TOF analysis with the use of sodium

5 cationic agents.

6 Acknowledgements

7 The authors wish to thank the National Council of Science and Technology (CONACyT) for the 8 support provided through the project number 256727 and the Laboratorio Nacional de Materiales 9 Grafénicos (LNMG) of Centro de Investigación en Química Aplicada (CIQA) for the support with 10 the instrumentation of MALDI-TOF. The authors also thank Dr. Geraldina Rodriguez for her valuable 11 support during MALDI-TOF measurements.

\section{References}

14 [1] A.C. Crecelius, C.R. Becer, K. Knop, U.S. Schubert, Block length determination of the block

[2] B. Karolewicz, A review of polymers as multifunctional excipients in drug dosage form

[3] L.A.L. Fliervoet, M. Naja, M. Hembury, T. Vermonden, Heterofunctional Poly (ethylene glycol) (PEG) Macroinitiator Enabling Controlled Synthesis of ABC Triblock Copolymers, Macromolecules, 50 (2017) 8390-8397.

A. Krieg, C. Pietsch, A. Baumgaertel, M.D. Hager, C. Remzi, U.S. Schubert, Dual hydrophilic polymers based on (meth)acrylic acid and poly(ethylene glycol) - synthesis and water uptake behavior, Polym. Chem., 1, 2010, 1669-1676.

L. Ahmadkhani, M. Abbasian, A. Akbarzadeh, Synthesis of sharply thermo and PH responsive PMA-b-PNIPAM-b-PEGB-PNIPAM-b-PMA by RAFT radical polymerization and its schizophrenic micellization in aqueous solutions, Des. Monomers Polym. 20 (2017) 406-418.

6] Y. Han, S. Liu, H. Mao, L. Tian, W. Ning, Synthesis of Novel Temperature- and pH-Sensitive ABA Triblock Copolymers P(DEAEMA-co-MEO2MA-co-OEGMA)-b-PEG-b-P (DEAEMA-co-MEO2MA-co-OEGMA): Micellization, Sol-Gel Transitions, and Sustained BSA Release, Polymers, 8, (2016), 367.

[7] H. Cao, C. Chen, D. Xie, X. Chen, P. Wang, Y. Wang, H. Song, W. Wang, A hyperbranched 
1 amphiphilic acetal polymer for pH-sensitive drug delivery, Polym. Chem., 9, (2018), 1692177.

3 [8] W.K. Storms-Miller, C. Pugh, Prop-2-yn-1-yl 2-Bromo-2-methylpropanoate: Identification

[9] P. Krys, K. Matyjaszewski, Kinetics of Atom Transfer Radical Polymerization, Eur. Polym.

[10] Y. Wang, K. Matyjaszewski, Catalytic Halogen Exchange in Miniemulsion ARGET ATRP: A Pathway to Well-Controlled Block Copolymers, Macromol. Rapid Commun., (2020), 2000264.

[11] L. Charles, MALDI of synthetic polymers with labile end-groups, Mass Spectrometry Reviews 33 (2014), 523-543.

[12] Y. Li, J.N. Hoskins, S.G. Sreerama, M.A. Grayson, S.M. Grayson, The identification of synthetic homopolymer end groups and verification of their transformations using MALDITOF mass spectrometry, J. Mass. Spectrom., 45, (2010) 587-611.

[13] B. Zhang, H. Zhang, B.K. Myers, R. Elupula, J. Jayawickramarajah, S.M. Grayson, Determination of polyethylene glycol end group functionalities by combination of selective reactions and characterization by matrix assisted laser desorption/ionization time-of-flight mass spectrometry, Anal. Chim. Acta, 816, (2014) 28-40.

[14] B. B.K. Myers, B. Zhang, J.E. Lapucha, S.M. Grayson, The characterization of dendronized poly(ethylene glycol)s and poly(ethylene glycol) multi-arm stars using matrix-assisted laser desorption/ionization time-of-flight mass spectrometry, Anal. Chim. Acta, 808, (2014) 175189.

[15] W.S. H. Pasch, 2003. MALDI-TOF Mass Spectrometry of synthetic polymers., Springer., Berlin.

[16] R. Zenobi, R. Knochenmuss, Ion formation in MALDI mass spectrometry, Mass Spectrometry Reviews 17 (1999) 337-366.

[17] M. Karas, R. Kru, Ion Formation in MALDI: The Cluster Ionization Mechanism, Chem. Rev. 103 (2003). 427-439.

[18] Y. Li, J.N. Hoskins, S.G. Sreerama, S.M. Grayson, MALDI-TOF Mass Spectral Characterization of Polymers Containing an Azide Group: Evidence of Metastable Ions, Macromolecules, 43(14), (2010), 6225-6228.

[19] A.H. Soeriyadi, M.R. Whittaker, C. Boyer, T.P. Davis, Soft Ionization Mass Spectroscopy: Insights Into the Polymerization Mechanism, Polymer Chemistry, 51, (2013), 1475-1505. 
[20] M.E. Payne, S.M. Grayson, Characterization of Synthetic Polymers via Matrix Assisted Laser Desorption Ionization Time of Flight ( MALDI-TOF ) Mass Spectrometry, J. Vis. Exp. (136), (2018) 1-15.

[21] J. De Winter, G. Deshayes, F. Boon, O. Coulembier, P. Dubois, P. Gerbaux, MALDI-ToF analysis of polythiophene: Use of trans-2-[3-(4-t-butyl-phenyl)- 2-methyl-2-propenylidene] malononitrile - DCTB - as matrix, J. Mass Spectrom. 46 (2011) 237-246.

[22] E. Lallana, T. Ferreri, S.C. Carroccio, A.M. Puga and N. Tirelli, End-group rearrangements in poly(propylene sulfide) matrix assisted laser desorption/ionization time-of-flight analysis. Experimental evidence and possible mechanisms. Rapid Commun. Mass Spectrom., 26, (2012), 2158-2164.

[23] C.D. Borman, A.T. Jackson, A. Bunn, A.L. Cutter, D.J. Irvine, Evidence for the low thermal stability of poly(methyl methacrylate) polymer produced by atom transfer radical polymerisation, Polymer, 41, (2000), 6015-6020.

[24] V. Coessens, K. Matyjaszewski, End group transformation of polymers prepared by ATRP, substitution to azides, J. Macromol Sci. Pure Appl Chem., A36 (1999), 667-669.

[25] H. Keul, A. Neumann, B. Reining, H. Höcker, Synthesis of telechelic and block copolymers via "living" radical polymerization, Macromol. Sym. 161, (2000) 63-72.

[26] J. Couthouis, H. Keul, M. Möller, MALDI-TOF Analysis of Halogen Telechelic Poly(methyl methacrylate)s and Poly(methyl acrylate)s Prepared by Atom Transfer Radical Polymerization (ATRP) or Single Electron Transfer-Living Radical Polymerization (SET-LRP), Macromol. Chem. Phys. 216, (2015) 1791-1800.

[27] A. Tintaru, C. Chendo, T.N.T. Phan, M. Rollet, L. Giordano, End-Group Cleavage in MALDI of ATRP-Made Polystyrene: A Silver- Catalyzed Reaction during Sample Preparation, Anal. Chem. 85 (2013) 5454-5462.

[28] K. Kim, A. Hasneen, H.J. Paik, T. Chang, MALDI-TOF MS characterization of polystyrene synthesized by ATRP, Polym. 54 (2013) 6133-6139.

[29] E. Le Grognec, S. Gabriel, V. Uni, D. Bourgogne, B. V Gabriel, D. Chimie, L. Cpe-cnrs, V. Boule, Radical Polymerization of Styrene Controlled by Half-Sandwich Mo (III)/ Mo (IV) Couples: All Basic Mechanisms Are Possible, J. Am. Chem. Soc., 123 (2001) 9513-9524.

[30] C. Ladavière, P. Lacroix-Desmazes, F. Delolme, First Systematic MALDI/ESI Mass Spectrometry Comparison to Characterize Polystyrene Synthesized by Different Controlled Radical Polymerizations. Macromolecules 42 (2009) 70-84.

[31] O. Altintas, T. Josse, M. Abbasi, J. De Winter, V. Trouillet, P. Gerbaux, M. Wilhelm, C. Barner-Kowollik, ATRP-based polymers with modular ligation points under thermal and 
thermomechanical stress, Polym. Chem. 6 (2015) 2854-2868.

[32] O. Guven, Size Exclusion Chromatography of poly(ethylene glycol), British Polymer Journal, 18, (1986), 391-393.

[33] R. Knochenmuss, V. Karbach, U. Wiesli, K. Breuker, R. Zenobi, The Matrix Suppression Effect in Matrix-assisted Laser Desorption/Ionization: Application to Negative Ions and Further Characteristics, Rapid Commun. Mass Spectrom. 12 (1998) 529-534.

[34] K. Matyjaszewski, S.M. Jo, H.J. Paik, D.A. Shipp, An investigation into the CuX/2,2'Bipyridine $(\mathrm{X}=\mathrm{Br}$ or $\mathrm{Cl})$ mediated atom transfer radical polymerization of acrylonitrile, Macromolecules 32 (1999) 6431-6438.

[35] J. Kemptner, M. Marchetti-Deschmann, J. Siekmann, P.L. Turecek, H. Peter, G. Allmaier, Journal of Pharmaceutical and Biomedical Analysis GEMMA and MALDI-TOF MS of reactive PEGs for pharmaceutical applications, Journal of Pharmaceutical and Biomedical Analysis 52 (2010) 432-437.

[36] P.C. Kooijman, S. Kok, M. Honing, Independent assessment of matrix - assisted laser desorption / ionization mass spectrometry (MALDI-MS) sample preparation quality: Effect of sample preparation on MALDI - MS of synthetic polymers Rationale, Rapid Communications in Mass Spectrometry 31 (2017) 1-31.

[37] C. Barner-Kowollik, T. Gruendling, J. Falkenhagen, T. Robert, A.R. Michael, 2012. Mass Spectrometry in Polymer Chemistry. Weinheim, Germany: Wiley.

[38] H. Y. Lin; Y. A. Dyakov; Y. T. Lee, and C. K. Ni Temperature Dependence of Desorbed Ions and Neutrals and Ionization Mechanism of Matrix-Assisted Laser Desorption/Ionization. J. Am. Soc. Mass Spectrometry 2020 https://doi.org/10.1021/jasms.0c00101 